\title{
Axioms for a local Reidemeister trace in fixed point and coincidence theory on differentiable manifolds
}

Christopher P. Staecker

Fairfield University, cstaecker@fairfield.edu

Follow this and additional works at: https://digitalcommons.fairfield.edu/mathandcomputersciencefacultypubs

Copyright 2009 Springer, Journal of Fixed Point Theory and Applications

\section{Peer Reviewed}

\section{Repository Citation}

Staecker, Christopher P., "Axioms for a local Reidemeister trace in fixed point and coincidence theory on differentiable manifolds" (2009). Mathematics Faculty Publications. 31.

https://digitalcommons.fairfield.edu/mathandcomputerscience-facultypubs/31

\section{Published Citation}

Staecker, P. Christopher, Axioms for a local Reidemeister trace in fixed point and coincidence theory on differentiable manifolds. Journal of Fixed Point Theory and Applications 5, 2009, 237-247.

This Article is brought to you for free and open access by the Mathematics Department at DigitalCommons@Fairfield. It has been accepted for inclusion in Mathematics Faculty Publications by an authorized administrator of DigitalCommons@Fairfield. For more information, please contact digitalcommons@fairfield.edu. 


\title{
Axioms for a local Reidemeister trace in fixed point and coincidence theory on differentiable manifolds
}

\author{
P. Christopher Staecker
}

May 9, 2008

\begin{abstract}
We give axioms which characterize the local Reidemeister trace for orientable differentiable manifolds. The local Reidemeister trace in fixed point theory is already known, and we provide both uniqueness and existence results for the local Reidemeister trace in coincidence theory.
\end{abstract}

\section{Introduction}

The Reidemeister trace is a fundamental invariant in topological fixed point theory, generalizing both the Lefschetz and Nielsen numbers. It was originally defined by Reidemeister in [11. A more modern treatment, under the name "generalized Lefschetz number," was given by Husseini in [9].

If $X$ is a finite connected CW-complex with universal covering space $\widetilde{X}$ and fundamental group $\pi$, then the cellular chain complex $C_{q}(\widetilde{X})$ is a free $\mathbb{Z} \pi$ module. If $f: X \rightarrow X$ is a cellular map and $\widetilde{f}: \widetilde{X} \rightarrow \widetilde{X}$ is a lift of $f$, then the induced map $\widetilde{f}_{q}: C_{q}(\widetilde{X}) \rightarrow C_{q}(\tilde{X})$ can be viewed as a matrix with entries in $\mathbb{Z} \pi$ (with respect to some chosen $\mathbb{Z} \pi$ basis for $C_{q}(\widetilde{X})$ ). We then define

$$
R T(f, \widetilde{f})=\sum_{q=0}^{\infty}(-1)^{q} \rho\left(\operatorname{tr}\left(\widetilde{f}_{q}\right)\right),
$$

where tr is the sum of the diagonal entries of the matrix, and $\rho$ is the projection into the "Reidemeister classes" of $\pi$. The Reidemeister trace, then, is an element of $\mathbb{Z} R$, where $R$ is the set of Reidemeister classes. Wecken, in [13], proved what we will refer to as the Wecken Trace Theorem, that

$$
R T(f, \widetilde{f})=\sum_{[\alpha] \in R} \operatorname{ind}([\alpha])[\alpha],
$$

where ind $([\alpha])$ is the index of the Nielsen fixed point class associated to $[\alpha]$ (see e.g. [10]). Thus the number of terms appearing in the Reidemeister trace with 
nonzero coefficient is equal to the Nielsen number of $f$, and by the LefschetzHopf Theorem, the sum of the coefficients is equal to the Lefschetz number of $f$.

Recent work of Furi, Pera, and Spadini in [6] has given a new proof of the uniqueness of the fixed point index on orientable manifolds with respect to three natural axioms. In [12 their approach was extended to the coincidence index. The result is the following theorem:

Theorem 1. Let $X$ and $Y$ be oriented differentiable manifolds of the same dimension. The coincidence index $\operatorname{ind}(f, g, U)$ of two mappings $f, g: X \rightarrow Y$ over some open set $U \subset X$ is the unique integer-valued function satisfying the following axioms:

- (Additivity) If $U_{1}$ and $U_{2}$ are disjoint open subsets of $U$ whose union contains all coincidence points of $f$ and $g$ on $U$, then

$$
\operatorname{ind}(f, g, U)=\operatorname{ind}\left(f, g, U_{1}\right)+\operatorname{ind}\left(f, g, U_{2}\right) .
$$

- (Homotopy) If $f$ and $g$ are "admissably homotopic" to $f^{\prime}$ and $g^{\prime}$, then

$$
\operatorname{ind}(f, g, U)=\operatorname{ind}\left(f^{\prime}, g^{\prime}, U\right)
$$

- (Normalization) If $L(f, g)$ denotes the coincidence Lefschetz number of $f$ and $g$, then

$$
\operatorname{ind}(f, g, X)=L(f, g) .
$$

In the spirit of the above theorem, we demonstrate the existence and uniqueness of a local Reidemeister trace in coincidence theory subject to five axioms. A local Reidemeister trace for fixed point theory was given by Fares and Hart in [5], but no Reidemeister trace (local or otherwise) has appeared in the literature for coincidence theory.

We note that recent work by Gonçalves and Weber in [8] gives axioms for the Reidemeister trace in fixed point theory using entirely different methods. Their work uses no locality properties, and is based on axioms for the Lefschetz number by Arkowitz and Brown in [1].

In Section 2 we present our axiom set, and we prove the uniqueness in coincidence theory in Section 3 In the special case of local fixed point theory, we can obtain a slightly stronger uniqueness result which we discuss in Section 4. Section 5 is a demonstration of the existence in the setting of coincidence theory.

This paper contains pieces of the author's doctoral dissertation. The author would like to thank his dissertation advisor Robert F. Brown for assistance with both the dissertation work and with this paper. The author would also like to thank Peter Wong, who guided the early dissertation work and interested him in the coincidence Reidemeister trace. 


\section{The Axioms}

Throughout the paper, unless otherwise stated, let $X$ and $Y$ denote connected orientable differentiable manifolds of the same dimension. All maps $f, g: X \rightarrow$ $Y$ will be assumed to be continuous. The universal covering spaces of $X$ and $Y$ will be denoted $\widetilde{X}$ and $\widetilde{Y}$ with projection maps $p_{X}: \widetilde{X} \rightarrow X$ and $p_{Y}: \widetilde{Y} \rightarrow Y$. A lift of some map $f: X \rightarrow Y$ is a map $\widetilde{f}: \widetilde{X} \rightarrow \widetilde{Y}$ with $p_{Y} \circ \widetilde{f}=f \circ p_{X}$.

Let $f, g: X \rightarrow Y$ be maps, with induced homomorphisms $\phi, \psi: \pi_{1}(X) \rightarrow$ $\pi_{1}(Y)$ respectively. We will view elements of $\pi_{1}(X)$ and $\pi_{1}(Y)$ as covering transformations, so that for any $\widetilde{x} \in \widetilde{X}$ and $\sigma \in \pi_{1}(X)$, we have $\widetilde{f}(\sigma \widetilde{x})=$ $\phi(\sigma) \widetilde{f}(\widetilde{x})$ and $\widetilde{g}(\sigma \widetilde{x})=\psi(\sigma) \widetilde{g}(\widetilde{x})$.

We will partition the elements of $\pi_{1}(Y)$ into equivalence classes defined by the "doubly twisted conjugacy" relation:

$$
\alpha \sim \beta \Longleftrightarrow \alpha=\psi(\sigma)^{-1} \beta \phi(\sigma) .
$$

The equivalence classes with respect to this relation (denoted e.g. $[\alpha]$ ) are called Reidemeister classes. The set of Reidemeister classes is denoted $\mathcal{R}[f, g]$.

For any set $S$, let $\mathbb{Z} S$ denote the free abelian group generated by $S$, whose elements we write as sums of elements of $S$ with integer coefficients. For any such abelian group, there is a homomorphism $c: \mathbb{Z} S \rightarrow \mathbb{Z}$ defined as the sum of the coefficients:

$$
c\left(\sum_{i} k_{i} s_{i}\right)=\sum_{i} k_{i}
$$

for $s_{i} \in S$ and $k_{i} \in \mathbb{Z}$, and $i$ ranging over a finite set.

For some maps $f, g: X \rightarrow Y$ and an open subset $U \subset X$, let

$$
\operatorname{Coin}(f, g, U)=\{x \in U \mid f(x)=g(x)\} .
$$

We say that the triple $(f, g, U)$ is admissable if $\operatorname{Coin}(f, g, U)$ is compact. Two triples $(f, g, U)$ and $\left(f^{\prime}, g^{\prime}, U\right)$ are admissably homotopic if there is some pair of homotopies $F_{t}, G_{t}: X \times[0,1] \rightarrow X$ of $f, g$ to $f^{\prime}, g^{\prime}$ with $\{(x, t) \in U \times[0,1] \mid$ $\left.F_{t}(x)=G_{t}(x)\right\}$ compact.

Let $\mathcal{C}(X, Y)$ be the set of admissable tuples, all tuples of the form $(f, \tilde{f}, g, \widetilde{g}, U)$ where $f, g: X \rightarrow Y$ are maps, $(f, g, U)$ is an admissable triple, and $\widetilde{f}$ and $\widetilde{g}$ are lifts of $f$ and $g$.

Let $(f, \widetilde{f}, g, \widetilde{g}, U),\left(f^{\prime}, \widetilde{f}^{\prime}, g^{\prime}, \widetilde{g}^{\prime}, U\right) \in \mathcal{C}(X, Y)$ with $(f, g, U)$ admissably homotopic to $\left(f^{\prime}, g^{\prime}, U\right)$ by homotopies $F_{t}, G_{t}$. By the homotopy lifting property, there are unique lifted homotopies $\widetilde{F}_{t}, \widetilde{G}_{t}: \widetilde{X} \times[0,1] \rightarrow \widetilde{Y}$ with $\widetilde{F}_{0}=\widetilde{f}$ and $\widetilde{G}_{0}=\widetilde{g}$. If we additionaly have $\widetilde{F}_{1}=\widetilde{f}^{\prime}$ and $\widetilde{G}_{1}=\widetilde{g}^{\prime}$, then we say that the tuples $(f, \widetilde{f}, g, \widetilde{g}, U)$ and $\left(f^{\prime}, \widetilde{f}^{\prime}, g^{\prime}, \widetilde{g}^{\prime}, U\right)$ are admisssably homotopic.

Throughout the following, let $R T$ be any function which to an admissable tuple $(f, \widetilde{f}, g, \widetilde{g}, U) \in \mathcal{C}(X, Y)$ associates an element of $\mathbb{Z} \mathcal{R}[f, g]$. Our first three axioms for the local Reidemeister trace are modeled after the axioms of Theorem 1. 
Axiom 1 (Additivity). Given $(f, \tilde{f}, g, \widetilde{g}, U) \in \mathcal{C}(X, Y)$, if $U_{1}$ and $U_{2}$ are disjoint open subsets of $U$ with $\operatorname{Coin}(f, g, U) \subset U_{1} \cup U_{2}$, then

$$
R T(f, \tilde{f}, g, \widetilde{g}, U)=R T\left(f, \tilde{f}, g, \widetilde{g}, U_{1}\right)+R T\left(f, \tilde{f}, g, \widetilde{g}, U_{2}\right) .
$$

Axiom 2 (Homotopy). If $(f, \tilde{f}, g, \widetilde{g}, U)$ and $\left(f^{\prime}, \widetilde{f}^{\prime}, g^{\prime}, \widetilde{g}^{\prime}, U\right)$ are admissably homotopic admissable tuples, then

$$
R T(f, \tilde{f}, g, \widetilde{g}, U)=R T\left(f^{\prime}, \widetilde{f}^{\prime}, g^{\prime}, \widetilde{g}^{\prime}, U\right) .
$$

Axiom 3 (Normalization). If $(f, \tilde{f}, g, \widetilde{g}, X) \in \mathcal{C}(X, Y)$, then

$$
c(R T(f, \tilde{f}, g, \widetilde{g}, X))=L(f, g),
$$

where $L(f, g)$ is the Lefschetz number of $f$ and $g$.

We will require one additional axiom to make some connections with Nielsen theory, based on a well-known property of the Reidemeister trace:

Axiom 4 (Lift invariance). For any $(f, \widetilde{f}, g, \widetilde{g}, U) \in \mathcal{C}(X, Y)$, and any $\alpha, \beta \in$ $\pi_{1}(Y)$ we have

$$
c(R T(f, \widetilde{f}, g, \widetilde{g}, U))=c(R T(f, \alpha \widetilde{f}, g, \beta \widetilde{g}, U)) .
$$

The four axioms above are enough to demonstrate some relationships between $R T$ and the coincidence index.

Proposition 1. If RT satisfies the homotopy, additivity, normalization, and lift invariance axioms, then

$$
c(R T(f, \tilde{f}, g, \widetilde{g}, U))=\operatorname{ind}(f, g, U)
$$

for any $(f, \widetilde{f}, g, \widetilde{g}, U) \in \mathcal{C}(X, Y)$, where ind denotes the coincidence index (see [7]).

Proof. Let $\omega=c \circ R T: \mathcal{C}(X, Y) \rightarrow \mathbb{Z}$. By the lift invariance axiom, $\omega$ is independent of the choice of lifts. Thus $\omega$ can be viewed as a function from the set of all admissable triples to $\mathbb{Z}$. It is clear that $\omega$ satisfies the three axioms of Theorem 1, since they are implied by our additivity, homotopy, and normalization axioms for $R T$ (disregarding the lift parameters). Thus $\omega$ is the coincidence index.

Proposition 2. If RT satisfies the additivity, homotopy, normalization, and lift invariance axioms and $c(R T(f, \widetilde{f}, g, \widetilde{g}, U)) \neq 0$, then there is some $\sigma \in \pi_{1}(Y)$ such that $\sigma \widetilde{f}$ and $\widetilde{g}$ have a coincidence on $p_{X}^{-1}(U)$.

Proof. By Proposition 1 if $c(R T(f, \tilde{f}, g, \widetilde{g}, U)) \neq 0$ then $\operatorname{ind}(f, g, U) \neq 0$, and so $f$ and $g$ have a coincidence on $U$. Let $x \in U$ be this coincidence point, and choose $\widetilde{x} \in p_{X}^{-1}(x)$. Then since $\widetilde{f}$ and $\widetilde{g}$ are lifts, the points $\widetilde{f}(\widetilde{x})$ and $\widetilde{g}(\widetilde{x})$ will project to the same point of $Y$ by $p_{Y}$. Thus there is some covering transformation $\sigma$ with $\sigma \widetilde{f}(\widetilde{x})=\widetilde{g}(\widetilde{x})$. 
The four axioms given above are not sufficient to uniquely characterize the Reidemeister trace in fixed point or coincidence theory. For instance, the function defined by

$$
T(f, \widetilde{f}, g, \widetilde{g}, U)=\operatorname{ind}(f, g, U)[1],
$$

where [1] is the Reidemeister class of the trivial element $1 \in \pi_{1}(Y)$, satisfies all of the axioms above, but provides none of the expected data concerning $\mathcal{R}[f, g]$, and so that function cannot be the Reidemeister trace.

An additional axiom is needed, one which somehow indicates the elements of $\mathcal{R}[f, g]$ which are to appear in the Reidemeister trace. Our final axiom is a sort of strengthening of Proposition 2, which specifies the Reidemeister data associated to the coincidence points.

Axiom 5 (Coincidence of lifts). If $[\alpha]$ appears with nonzero coefficient in $R T(f, \widetilde{f}, g, \widetilde{g}, U)$, then $\alpha \widetilde{f}$ and $\widetilde{g}$ have a coincidence on $p_{X}^{-1}(U)$.

Any function $R T$ which to a tuple $(f, \widetilde{f}, g, \widetilde{g}, U) \in \mathcal{C}(X, Y)$ associates an element of $\mathbb{Z} \mathcal{R}[f, g]$, and satisfies the additivity, homotopy, normalization, lift invariance, and coincidence of lifts axioms we will call a local Reidemeister trace. Our main result (Theorem 3) states that there is a unique such function.

\section{$3 \quad$ Uniqueness}

Let $(f, \widetilde{f}, g, \widetilde{g}, U) \in \mathcal{C}(X, Y)$, let $\widetilde{U}=p_{X}^{-1}(U)$, and let

$$
C(\widetilde{f}, \widetilde{g}, \widetilde{U},[\alpha])=p_{X}(\operatorname{Coin}(\alpha \tilde{f}, \widetilde{g}, \widetilde{U})) .
$$

For each $\alpha$ we have $C(\widetilde{f}, \widetilde{g}, \widetilde{U},[\alpha]) \subset \operatorname{Coin}(f, g, U)$, and such coincidence sets are called coincidence classes. That these classes are well defined is a consequence of the following lemma, which appears in slightly different language as Lemma 2.3 of $[4$.

Lemma 1. Let $\alpha, \beta \in \pi_{1}(Y)$, maps $f, g: X \rightarrow Y$, and an open subset $U \subset X$ be given. Then:

- $[\alpha]=[\beta]$ if and only if

$$
p_{X} \operatorname{Coin}(\alpha \tilde{f}, \widetilde{g}, \widetilde{U})=p_{X} \operatorname{Coin}(\beta \tilde{f}, \widetilde{g}, \widetilde{U})
$$

for any lifts $\widetilde{f}, \widetilde{g}$.

- If $[\alpha] \neq[\beta]$, then $p_{X} \operatorname{Coin}(\alpha \widetilde{f}, \widetilde{g}, \widetilde{U})$ and $p_{X} \operatorname{Coin}(\alpha \widetilde{f}, \widetilde{g}, \widetilde{U})$ are disjoint for any lifts $\tilde{f}, \widetilde{g}$.

Given the above notation, the coincidence of lifts axiom could be restated as follows: If $[\alpha]$ appears with nonzero coefficient in $R T(f, \widetilde{f}, g, \widetilde{g}, U)$, then $C(\widetilde{f}, \widetilde{g}, \widetilde{U},[\alpha])$ is nonempty. For each coincidence point $x$ in $U$, define $\left[x_{\widetilde{f}, \widetilde{g}}\right] \in$ $\mathcal{R}[f, g]$ as that class $[\alpha]$ for which $x \in C(\widetilde{f}, \widetilde{g}, \widetilde{U},[\alpha])$. 
Theorem 2. If RT is a local Reidemeister trace and $\operatorname{Coin}(f, g, U)$ is a set of isolated points, then

$$
R T(f, \tilde{f}, g, \widetilde{g}, U)=\sum_{x \in \operatorname{Coin}(f, g, U)} \operatorname{ind}\left(f, g, U_{x}\right)\left[x_{\tilde{f}, \widetilde{g}}\right],
$$

where $U_{x}$ is an isolating neighborhood for the coincidence point $x$.

Proof. By the additivity property, we need only show that

$$
R T\left(f, \widetilde{f}, g, \widetilde{g}, U_{x}\right)=\operatorname{ind}\left(f, g, U_{x}\right)\left[x_{\widetilde{f}, \widetilde{g}}\right] .
$$

First, we observe that no element of $\mathcal{R}[f, g]$ other than $\left[x_{\tilde{f}, \tilde{g}}\right]$ appears as a term with nonzero coefficient in $R T\left(f, \widetilde{f}, g, \widetilde{g}, U_{x}\right)$ : If some $[\beta]$ does appear with nonzero coefficient, then we know by the coincidence of lifts axiom that $\beta \tilde{f}$ and $\widetilde{g}$ have a coincidence on $\widetilde{U}_{x}=p_{X}^{-1}\left(U_{x}\right)$. Projection of this coincidence point gives a coincidence point in $U_{x}$ which necessarily must be $x$, since $x$ is the only coincidence point in $U_{x}$. Thus $x \in p_{X} \operatorname{Coin}\left(\beta \widetilde{f}, \widetilde{g}, \widetilde{U}_{x}\right)$, which means that $[\beta]=\left[x_{\widetilde{f}, \widetilde{g}}\right]$. have

Since $\left[x_{\widetilde{f}, \widetilde{g}}\right]$ is the only element of $\mathcal{R}[f, g]$ appearing in $R T(f, \widetilde{f}, g, \widetilde{g}, U)$, we

$$
R T\left(f, \tilde{f}, g, \widetilde{g}, U_{x}\right)=k\left[x_{\widetilde{f}, \widetilde{g}}\right]
$$

for some $k \in \mathbb{Z}$ (possibly $k=0$ ). Proposition 1 says that the coefficient sum must equal the index, and so $k=\operatorname{ind}\left(f, g, U_{x}\right)$ as desired.

The above is a strong result for maps whose coincidence sets are isolated. In order to leverage this result for arbitrary maps, we will make use of a technical lemma, a combination of Lemmas 13 and 15 from [12.

Lemma 2. Let $(f, g, U)$ be an admissable triple, and let $V \subset U$ be an open subset containing $\operatorname{Coin}(f, g, U)$ with compact closure $\bar{V} \subset U$. Then $(f, g, V)$ is admissably homotopic to an admissable triple $\left(f^{\prime}, g^{\prime}, V\right)$, where $f^{\prime}$ and $g^{\prime}$ have isolated coincidence points in $V$.

The above lemma is used to approximate any maps by maps having isolated coincidence points, and we obtain our uniqueness theorem:

Theorem 3. There is at most one local Reidemeister trace defined on $\mathcal{C}(X, Y)$.

Proof. Let $R T$ be local Reidemeister trace, and take $(f, \widetilde{f}, g, \widetilde{g}, U) \in \mathcal{C}(X, Y)$. Then by Lemma 2 there is an open subset $V \subset U$ with $\operatorname{Coin}(f, U) \subset V$ and maps $f^{\prime}, g^{\prime}$ with isolated coincidence points with $(f, g, V)$ admissably homotopic to $\left(f^{\prime}, g^{\prime}, V\right)$. Then by the homotopy axiom there are lifts $\widetilde{f}^{\prime}, \widetilde{g}^{\prime}$ of $f$ and $g$ with

$$
R T(f, \widetilde{f}, g, \widetilde{g}, U)=R T\left(f^{\prime}, \widetilde{f}^{\prime}, g^{\prime}, \widetilde{g}^{\prime}, V\right) .
$$

The coincidence points of $f^{\prime}$ and $g^{\prime}$ in $V$ are isolated, so we have

$$
R T(f, \widetilde{f}, g, \widetilde{g}, U)=\sum_{x \in \operatorname{Coin}\left(f^{\prime}, g^{\prime}, V\right)} \operatorname{ind}\left(f^{\prime}, g^{\prime}, V_{x}\right)\left[x_{\widetilde{f}^{\prime}, \widetilde{g}^{\prime}}\right]
$$


where $V_{x}$ is an isolating neighborhood of the coincidence point $x$. This gives an explicit formula for the computation of $R T(f, \widetilde{f}, g, \widetilde{g}, U)$. The only choice made in the computation is of the admissable homotopy to $\left(f^{\prime}, g^{\prime}, V\right)$, but any alternative choice must give the same local Reidemeister trace by the homotopy axiom. Thus all local Reidemeister traces must be computed in the same way, giving the same result, which means that there can be only one.

\section{Uniqueness in fixed point theory}

In the special case where $Y=X$ and $g$ is taken to be the identity map id : $X \rightarrow X$, the above method can be used with slight modifications to prove a uniqueness result for the local Reidemeister trace in the fixed point theory of possibly nonorientable manifolds.

We have not in this paper made explicit use of the orientability hypothesis, but it is a necessary hypothesis for the uniqueness of the coincidence index in Theorem 1. which was used in Proposition 1. An accounting of orientations is needed in coincidence theory to distinguish between points of index +1 and index -1 (though see [4 for an approach to an index for nonorientable manifolds, which does not always give an integer). Orientability is not needed in local fixed point theory, since the notion of an orientation preserving selfmap is well-defined locally, even on a manifold with no global orientation. Thus the uniqueness of the fixed point index in [6] does not require orientability, and we will not require it here.

Let $\mathcal{C}(X)$ be the set of all tuples of the form $(f, \tilde{f}, \widetilde{\imath}, U)$, where $f: X \rightarrow X$ is a selfmap, $\widetilde{f}: \widetilde{X} \rightarrow \widetilde{X}$ is a lift of $f$, the map $\widetilde{\imath}: \widetilde{X} \rightarrow \widetilde{X}$ is a lift of the identity map, and $U$ is an open subset of $X$ with compact fixed point set $\operatorname{Fix}(f, U)=\operatorname{Coin}(f$, id, $U)$. Let $\mathcal{R}[f]=\mathcal{R}[f$, id $]$. Two tuples $(f, \widetilde{f}, \widetilde{\imath}, U)$ and $\left(f^{\prime}, \widetilde{f}^{\prime}, \widetilde{\imath}, U\right)$ are said to be admissably homotopic if there is some homotopy $F_{t}$ of $f$ to $f^{\prime}$ with $\left\{(x, t) \mid F_{t}(x)=x\right\}$ compact, and $F_{t}$ lifts to a homotopy of $\widetilde{f}$ to $\tilde{f}^{\prime}$.

Our uniqueness theorem is then:

Theorem 4. If $X$ is a (possibly nonorientable) differentiable manifold, then there is a unique function taking an admissable tuple $(f, \tilde{f}, \widetilde{\imath}, U)$ to an element of $\mathbb{Z} \mathcal{R}[f]$ satisfying the following axioms:

- (Additivity) If $U_{1}$ and $U_{2}$ are disjoint open subsets of $U$ with $\operatorname{Fix}(f, U) \subset$ $U_{1} \cup U_{2}$, then

$$
R T(f, \tilde{f}, \widetilde{\imath}, U)=R T\left(f, \widetilde{f}, \widetilde{\imath}, U_{1}\right)+R T\left(f, \tilde{f}, \widetilde{\imath}, U_{2}\right)
$$

- (Homotopy) If $(f, \widetilde{f}, \widetilde{\imath}, U)$ is admissably homotopic to $\left(f^{\prime}, \widetilde{f}^{\prime}, \widetilde{\imath}, U\right)$, then

$$
R T(f, \widetilde{f}, \widetilde{\imath}, U)=R T\left(f^{\prime}, \widetilde{f}^{\prime}, \widetilde{\imath}, U\right)
$$


- (Weak normalization) If $f$ is a constant map, then

$$
c(R T(f, \tilde{f}, \widetilde{\imath}, U))=1
$$

- (Lift invariance) For any $\alpha, \beta \in \pi_{1}(X)$, we have

$$
c(R T(f, \widetilde{f}, \widetilde{\imath}, U))=c(R T(f, \alpha \widetilde{f}, \beta \widetilde{\imath}, U))
$$

- (Coincidence of lifts) If $[\alpha]$ appears with nonzero coefficient in $R T(f, \widetilde{f}, \widetilde{\imath}, U)$, then $\alpha \widetilde{f}$ and $\widetilde{\imath}$ have a coincidence point on $p_{X}^{-1}(U)$.

Proof. First we note that a result analagous to Proposition 1 can be obtained in the fixed point setting using only the weak normalization axiom: Using the three axioms of [6], which make use of an appropriately weakened normalization axiom, we see that $c \circ R T$ is the fixed point index.

Then letting $g=$ id in the proof of Theorem 2, we have that, if $f$ has isolated fixed points,

$$
R T(f, \tilde{f}, \widetilde{\imath}, U)=\sum_{x \in \operatorname{Fix}\left(f, U_{x}\right)} \operatorname{ind}\left(f, U_{x}\right)\left[x_{\widetilde{f}, \tilde{\imath}}\right],
$$

where ind denotes the fixed point index, and $U_{x}$ is an isolating neighborhood for the fixed point $x$.

A fixed point version of Lemma 2 can be found in Lemmas 4.1 and 3.3 of [6], and the proof of Theorem [3 can be mimicked to obtain our uniqueness result.

Note that the uniqueness in fixed point theory requires only a weakened version of the normalization axiom. A uniqueness result for coincidence theory using only the weak normalization axiom can be obtained if we restrict ourselves to self-maps of a particular (not necessarily orientable) manifold. This would use a proof similar to the above, using results from Section 5 of [12].

\section{Existence}

The existence of a local Reidemeister trace in fixed point theory for connected finite dimensional locally compact polyhedra is established by Fares and Hart in [5]. There, the slightly more general local $H$-Reidemeister trace is defined, called "the local generalized $H$-Lefschetz number". An extension of this paper to the mod $H$ theory would not be difficult. The fact that the mod $H$ Reidemeister classes are unions of ordinary Reidemeister classes allows the same results to be obtained without substantial modifications.

In [5, the additivity and homotopy axioms are proved in Proposition 3.2.9 and Proposition 3.2.8, respectively. A strong version of the lift invariance axiom (see our Theorem 6) is proved in Proposition 3.2.4. The coincidence of lifts axiom is not stated explicitly by Fares and Hart, but is a straightforward consequence of their trace-like definition (if some $[\alpha]$ has nonzero coefficient in the 
Reidemeister trace, it neccesarily comes from some simplex in the covering space containing a fixed point of $\alpha \widetilde{f}$ ). A result analogous to the Wecken Trace Theorem (which trivially implies the normalization and weak normalization axioms) is given in Theorem 3.3.1.

No Reidemeister trace for coincidence theory, either local or global, has appeared previously in the literature. The proof of Theorem 3 furnishes the appropriate definition, as follows: Given an admissable tuple $(f, \widetilde{f}, g, \widetilde{g}, U)$, we find (by Lemma2) an admissably homotopic tuple $\left(f^{\prime}, \widetilde{f}^{\prime}, g^{\prime}, \widetilde{g}^{\prime}, V\right)$ with isolated coincidence points, and we define

$$
R T(f, \widetilde{f}, g, \widetilde{g}, U)=\sum_{x \in \operatorname{Coin}\left(f^{\prime}, g^{\prime}, V\right)} \operatorname{ind}\left(f^{\prime}, g^{\prime}, V_{x}\right)\left[x_{\widetilde{f}^{\prime}, \widetilde{g}^{\prime}}\right],
$$

where $V_{x}$ is an isolating neighborhood for the coincidence point $x$. The above is well defined provided that it is independent of the choice of the admissably homotopic tuple. This is ensured by the following lemma:

Lemma 3. If $(f, \widetilde{f}, g, \widetilde{g}, U)$ and $\left(f^{\prime}, \widetilde{f}^{\prime}, g^{\prime}, \widetilde{g}^{\prime}, U\right)$ are admissably homotopic tuples with isolated coincidence points, then

$$
\sum_{x \in \operatorname{Coin}(f, g, U)} \operatorname{ind}\left(f, g, U_{x}\right)\left[x_{\widetilde{f}, \widetilde{g}}\right]=\sum_{x^{\prime} \in \operatorname{Coin}\left(f^{\prime}, g^{\prime}, U\right)} \operatorname{ind}\left(f^{\prime}, g^{\prime}, U_{x^{\prime}}\right)\left[x_{\tilde{f}^{\prime}, \widetilde{g}^{\prime}}^{\prime}\right],
$$

where $U_{x}$ is an isolating neighborhood for the coincidence point $x \in \operatorname{Coin}(f, g, U)$, and $U_{x^{\prime}}$ is an isolating neighborhood of the coincidence point $x^{\prime} \in \operatorname{Coin}\left(f^{\prime}, g^{\prime}, U\right)$.

Proof. We define the index of a coincidence class $C$ of $f$ and $g$ as follows:

$$
\text { ind } C=\sum_{x \in C} \operatorname{ind}\left(f, g, U_{x}\right) \text {. }
$$

A class is called essential if its index is nonzero.

Since $f$ and $g$ are homotopic to $f^{\prime}$ and $g^{\prime}$, we have $\mathcal{R}[f, g]=\mathcal{R}\left[f^{\prime}, g^{\prime}\right]$. Call this common set of Reidemeister classes $R$. Letting $\widetilde{U}=p_{X}^{-1}(U)$, the statement of the Lemma is equivalent to

$$
\sum_{[\alpha] \in R} \operatorname{ind} C(\tilde{f}, \widetilde{g}, \widetilde{U},[\alpha])[\alpha]=\sum_{[\alpha] \in R} \operatorname{ind} C\left(\widetilde{f}^{\prime}, \widetilde{g}^{\prime}, \widetilde{U},[\alpha]\right)[\alpha],
$$

and we need only show that ind $C(\widetilde{f}, \widetilde{g}, \widetilde{U},[\alpha])=\operatorname{ind} C\left(\widetilde{f}^{\prime}, \widetilde{g}^{\prime}, \widetilde{U},[\alpha]\right)$ for any $[\alpha]$. We will prove this using Brooks's notion of homotopy-relatedness of coincidence classes, exposited in detail in 2] and briefly in [3].

Let $F_{t}, \widetilde{F}_{t}, G_{t}, \widetilde{G}_{t}$ be homotopies realizing the admissable homotopy of $(f, \widetilde{f}, g, \widetilde{g}, U)$ and $\left(f^{\prime}, \widetilde{f}^{\prime}, g^{\prime}, \widetilde{g}^{\prime}, U\right)$. Two coincidence points $x \in \operatorname{Coin}(f, g, U)$ and $x^{\prime} \in \operatorname{Coin}\left(f^{\prime}, g^{\prime}, U\right)$ are $\left(F_{t}, G_{t}\right)$-related if there is some path $\gamma(t)$ in $X$ connecting $x$ to $x^{\prime}$ such that the paths $F_{t}(\gamma(t))$ and $G_{t}(\gamma(t))$ are homotopic in $Y$ as paths with fixed endpoints. Two coincidence classes are related if at least one point of one is related 
to at least one point of the other. Theorem II.22 of [2] shows that the notion of $\left(F_{t}, G_{t}\right)$-relatedness gives a bijective correspondence between the essential coincidence classes of $(f, g)$ and those of $\left(f^{\prime}, g^{\prime}\right)$. Theorem IV.24 of [2] further shows that any two such related classes will have the same index.

What remains is an elementary argument using covering-space theory. Let $C=C(\widetilde{f}, \widetilde{g}, \widetilde{U},[\alpha])$, and let $C^{\prime}$ be the unique coincidence class of $\left(f^{\prime}, g^{\prime}\right)$ which is $\left(F_{t}, G_{t}\right)$-related to $C$. We need only show that $C^{\prime}=C\left(\widetilde{f}^{\prime}, \widetilde{g}^{\prime}, \widetilde{U},[\alpha]\right)$, and thus (since homotopy-relatedness preserves the index) that ind $C(\widetilde{f}, \widetilde{g}, \widetilde{U},[\alpha])=$ ind $C\left(\widetilde{f}^{\prime}, \widetilde{g}^{\prime}, \widetilde{U},[\alpha]\right)$.

Choose a point $x \in C$, and let $x^{\prime}$ be a point in $C^{\prime}$ which is $\left(F_{t}, G_{t}\right)$ related to $x$. Then there is some path $\gamma$ in $X$ from $x$ to $x^{\prime}$ with $F_{t}(\gamma(t))$ homotopic to $G_{t}(\gamma(t))$. Let $\widetilde{x}$ be some point with $p_{X}(\widetilde{x})=x$ and $\alpha \widetilde{f}(\widetilde{x})=\widetilde{g}(\widetilde{x})$. We can lift $\gamma$ to a path $\widetilde{\gamma}$ in $\widetilde{X}$ starting at $\widetilde{x}$. Since $F_{t}(\gamma(t))$ is homotopic to $G_{t}(\gamma(t))$, we will have $\widetilde{F}_{t}(\widetilde{\gamma}(t))$ homotopic to $\widetilde{G}_{t}(\widetilde{\gamma}(t))$, which in particular means that they will have the same endpoint. This common endpoint is $\alpha \widetilde{f}^{\prime}(\widetilde{\gamma}(1))=\widetilde{g}^{\prime}(\widetilde{\gamma}(1))$, which must project by $p_{X}$ to the point $x^{\prime}$. Thus $x^{\prime} \in p_{X}\left(\operatorname{Coin}\left(\alpha \widetilde{f}^{\prime}, \widetilde{g}^{\prime}, \widetilde{U}\right)\right)$, and so $C^{\prime}=C\left(\widetilde{f^{\prime}}, \widetilde{g}^{\prime}, \widetilde{U},[\alpha]\right)$, as desired.

We have thus produced a meaningful definition of a local coincidence Reidemeister trace on orientable differentiable manifolds of the same dimension, and the proof above suffices to give:

Theorem 5 (Wecken Coincidence Trace Theorem). Let RT be the unique local coincidence Reidemeister trace satisfying our five axioms. Then for any $(f, \widetilde{f}, g, \widetilde{g}, U) \in \mathcal{C}(X, Y)$ with $\widetilde{U}=p_{X}^{-1}(U)$, we have

$$
R T(f, \tilde{f}, g, \widetilde{g}, U)=\sum_{[\alpha] \in \mathcal{R}[f, g]} \operatorname{ind} C(\widetilde{f}, \widetilde{g}, \widetilde{U},[\alpha])[\alpha] .
$$

In conclusion we prove a stronger form of the lift invariance axiom, a coincidence version of a well-known property of the Reidemeister trace.

Theorem 6. Let RT be the unique local coincidence Reidemeister trace satisfying our five axioms. If

$$
R T(f, \tilde{f}, g, \widetilde{g}, U)=\sum_{[\sigma] \in \mathcal{R}[f, g]} k_{[\sigma]}[\sigma]
$$

for $k_{[\sigma]} \in \mathbb{Z}$, then for any $\alpha, \beta \in \pi_{1}(Y)$, we have

$$
R T(f, \alpha \widetilde{f}, g, \beta \widetilde{g}, U)=\sum_{[\sigma] \in \mathcal{R}[f, g]} k_{[\sigma]}\left[\beta \sigma \alpha^{-1}\right] .
$$

Proof. Letting $\widetilde{U}=p_{X}^{-1}(U)$, by Theorem $\left[\right.$ we know that $k_{[\sigma]}=\operatorname{ind} C(\widetilde{f}, \widetilde{g}, \widetilde{U},[\sigma])$. Then we have

$C(\alpha \widetilde{f}, \beta \widetilde{g},[\sigma])=p_{X} \operatorname{Coin}(\sigma \alpha \tilde{f}, \beta \widetilde{g}, \widetilde{U})=p_{X} \operatorname{Coin}\left(\beta^{-1} \sigma \alpha \widetilde{f}, \widetilde{g}, \widetilde{U}\right)=C\left(\widetilde{f}, \widetilde{g},\left[\beta^{-1} \sigma \alpha\right]\right)$, 
and thus ind $C(\alpha \widetilde{f}, \beta \widetilde{g}, \widetilde{U},[\sigma])=k_{\left[\beta^{-1} \sigma \alpha\right]}$. Now by Theorem [5] again, we have

$$
\begin{aligned}
R T(f, \alpha \tilde{f}, g, \beta \widetilde{g}, \widetilde{U}) & =\sum_{[\sigma] \in \mathcal{R}[f, g]} \text { ind } C(\alpha \tilde{f}, \beta \widetilde{g},[\sigma])[\sigma]=\sum_{[\sigma] \in \mathcal{R}[f, g]} k_{\left[\beta^{-1} \sigma \alpha\right]}[\sigma] \\
& =\sum_{[\gamma] \in \mathcal{R}[f, g]} k_{[\gamma]}\left[\beta \gamma \alpha^{-1}\right],
\end{aligned}
$$

as desired.

\section{References}

[1] M. Arkowitz and R. Brown. The Lefschetz-Hopf theorem and axioms for the Lefschetz number. Fixed Point Theory and Applications, 1:1-11, 2004.

[2] R. Brooks. Coincidences, Roots, and Fixed Points. Doctoral dissertation, University of California, Los Angeles, 1967.

[3] R. Brooks and R. Brown. A lower bound on the $\Delta$-Nielsen number. Transactions of the American Mathematical Society, 143:555-564, 1969.

[4] R. Dobreńko and J. Jezierski. The coincidence Nielsen number on nonorientable manifolds. The Rocky Mountain Journal of Mathematics, 23:67-85, 1993.

[5] J. Fares and E. Hart. A generalized Lefschetz number for local Nielsen fixed point theory. Topology and Its Applications, 59:1-23, 1994.

[6] M. Furi, M. P. Pera, and M. Spadini. On the uniqueness of the fixed point index on differentiable manifolds. Fixed Point Theory and Applications, $4: 251-259,2004$.

[7] D. L. Gonçalves. Coincidence theory. In R.F. Brown, editor, The Handbook of Topological Fixed Point Theory, pages 3-42. Springer, 2005.

[8] D. L. Gonçalves and J. Weber. Axioms for the equivariant Lefschetz number and for the Reidemeister trace. Journal of Fixed Point Theory and Applications, 2:55-72, 2007.

[9] S. Husseini. Generalized Lefschetz numbers. Transactions of the American Mathematical Society, 272:247-274, 1982.

[10] B. Jiang. Lectures on Nielsen fixed point theory. Contemporary Mathematics 14, American Mathematical Society, 1983.

[11] K. Reidemeister. Automorphismen von Homotopiekettenringen. Mathematische Annalen, 112:586-593, 1936.

[12] P. C. Staecker. On the uniqueness of the coincidence index on orientable differentiable manifolds. Topology and Its Applications, 154:1961-1970, 2007, arxiv eprint math.GN/0607751. 
[13] F. Wecken. Fixpunktklassen I, II, III. Mathematische Annalen, 117, 118:659-671, 216-234, 544-577, 1941, 1942. 